\title{
Anti-depressant-like effect of atractylenolide $I$ in a mouse model of depression induced by chronic unpredictable mild stress
}

\author{
HAIYAN GAO, XIANGHUA ZHU, YANG XI, QUN LI, ZHENZHONG SHEN and YONGJIE YANG \\ Department of Psychiatry, Xuzhou Oriental People's Hospital, Xuzhou, Jiangsu 221004, P.R. China
}

Received November 10, 2016; Accepted July 7, 2017

DOI: 10.3892/etm.2017.5517

\begin{abstract}
Atractylenolide I (AT-I), a major component of the rhizoma of Atractylodes macrocephala Koidz., exerts a wide range of activities. The purpose of the present study was to investigate the anti-depressant-like effect of AT-I in a mouse model of chronic unpredictable mild stress (CUMS), and to explore the possible molecular mechanism involved. It was revealed that AT-I significantly ameliorated CUMS-induced depressive-like behaviors, as evidenced by increased sucrose preference as well as shortened immobility time in the forced swimming and the tail suspension test. In addition, AT-I reduced CUMS-induced decreases in the concentrations of serotonin and norepinephrine in the hippocampus. Furthermore, AT-I inhibited the activation of the nucleotide binding and oligomerization domain-like receptor family pyrin domain-containing 3 (NLRP3) inflammasome as well as the concentration of the pro-inflammatory cytokine interleukin (IL)-1 $\beta$ in the hippocampi of mice subjected to CUMS. In conclusion, the results of the present study suggested that AT-I exerts anti-depressant-like effects in a CUMS-induced model of depression in mice, the molecular mechanism of which is associated with the inhibition of NLRP3 inflammasome activation to decrease IL- $1 \beta$ production.
\end{abstract}

\section{Introduction}

Depression affects an estimate of 350 million individuals worldwide (1) and is a serious public health problem due to its high morbidity, recurrence and suicide rate (2). Decades of extensive study of the pathophysiology of depression have led to various hypotheses regarding its molecular basis. Increasing evidence has demonstrated that depression is an inflammatory

Correspondence to: Dr Yongjie Yang, Department of Psychiatry, Xuzhou Oriental People's Hospital, 379 Tongshan Road, Yunlong, Xuzhou, Jiangsu 221004, P.R. China

E-mail: yyang4824@hotmail.com

Key words: depression, atractylenolide I, interleukin-1 $\beta$, nucleotide binding and oligomerization domain-like receptor family pyrin domain-containing 3 inflammasome, inflammation disorder (3). Clinical studies found that pro-inflammatory cytokines, including interleukin (IL)-1 $\beta$, IL-6 and tumor necrosis factor- $\alpha$ were significantly elevated during depression $(4,5)$. Bluthé et al (6) demonstrated that increased IL-1 $\beta$ levels are linked with depressive-like behavior in rats. The nucleotide binding and oligomerization domain-like receptor family pyrin domain-containing 3 (NLRP3) inflammasome, composed of NLRP3, apoptosis-associated speck-like protein (ASC) and caspase- 1 , is the major mediator of IL-1 $\beta$ transcription and function. Under stimulation and stress, the NLRP3 inflammasome complex induces the cleavage and activation of caspase-1, which promotes the maturation of IL-1 $\beta$ (7). A previous study reported that activated NLRP3 inflammasome along with increased serum levels of IL-1 $\beta$ and IL-18 were observed in mononuclear blood cells from patients with major depressive disorder (8). Furthermore, the NLRP3 inflammasome was demonstrated to mediate IL-1 $\beta$-associated central nervous system (CNS) inflammation in animal models of stress-induced depression $(9,10)$. All of the above suggested a key role of the NLRP3 inflammasome in the development of depression. Therefore, the NLRP3 inflammasome may be considered as a possible target for the prevention and treatment of depression.

Atractylenolide I (AT-I) is a major active component of the Rhizoma of Atractylodes macrocephala Koidz. and exerts a wide range of pharmacological activities, including anti-inflammatory $(11,12)$, anti-tumor $(13,14)$ and anti-angiogenic effects $(15)$. A previous study has implied a potential anti-depressive activity of AT-I (16). However, to date, the effect of AT-I in depression and the underlying molecular mechanisms have remained to be fully elucidated. Therefore, the present study aimed to investigate the anti-depressive like effect of AT-I in a mouse model of chronic unpredictable mild stress (CUMS). Behavioral tests, including the sucrose preference test (SPT), forced swim test (FST) and tail suspension test (TST) were performed to determine whether AT-I improves CUMS-induced depression in mice. Furthermore, the serum levels of the pro-inflammatory factor IL-1 $\beta$ as well as hippocampal serotonin (5-HT) and norepinephrine (NE) levels, and the expression levels of components of the NLRP3 inflammasome were assessed to explore the possible underlying molecular mechanisms.

\section{Materials and methods}

Animals. A total of 60 male ICR mice (weight, 18-20 g; age, 5 weeks old) were purchased from the Animal Center 
of Xuzhou Medicine University (Xuzhou, China). Animals were kept under a 12-h light/dark cycle at room temperature $\left(22 \pm 2^{\circ} \mathrm{C}\right.$; humidity, $\left.55 \pm 5 \%\right)$ with food and water provided ad libitum. All animal experiments were performed according to the Guidelines for the Care and Use of Laboratory Animals by the National Institute of Health. The study was approved by the ethics committee of Xuzhou Oriental People's Hospital (Xuzhou, China).

Mouse model of CUMS. The CUMS procedure was performed as previously described with slight modifications (17). In brief, stressors were applied for three weeks in a random order without repetition between weeks. The stimuli included $45^{\circ}$ cage tilt, tail pinching for $1 \mathrm{~min}$, cage shaking for $5 \mathrm{~min}$, swimming in $4^{\circ} \mathrm{C}$ cold water for $5 \mathrm{~min}$, reversed light/dark cycle, maintained in an empty squirrel cage, damp bedding, tail suspension, white noise, and $24 \mathrm{~h}$ of food and water deprivation (at the same time).

Experimental design. After adaption for one week, 48 male ICR mice were randomly divided into six groups (eight mice per group): Control group (unstressed + saline vehicle), model group (CUMS + saline vehicle), three AT-I treatment groups (CUMS + AT-I) and a fluoxetine group (CUMS + FLU). From the 4 th week, AT-I $(5,10$ or $20 \mathrm{mg} / \mathrm{kg})$ or fluoxetine $(20 \mathrm{mg} / \mathrm{kg})$ was daily administered by oral gavage for 3 weeks. AT-I was purchased from Sigma-Aldrich (Merck KGaA, Darmstadt, Germany) and fluoxetine hydrochloride was obtained from Changzhou Siyao Pharmaceuticals Co., Ltd. (Changzhou, China). After the last administration of AT-I or fluoxetine, behavioral tests were performed.

SPT. SPT was performed weekly following a previously described procedure (18). The sucrose preference value was calculated as follows: Preference value $(\%)=$ sucrose intake/(sucrose intake+water intake)x100\%.

FST. The FST was performed as described previously (19). The immobility time was defined as the duration for which the mouse floated in an upright position without struggling and making only slight movements to keep its head above the water.

TST. The TST was performed based on a previously described method (20). In brief, the mouse was suspended $25 \mathrm{~cm}$ above the floor by the tip of the tail $(1 \mathrm{~cm})$ tied up to the level. The immobility time was counted during a test period of 6 min (prior adaptation for $1 \mathrm{~min}$ and recording for the last $5 \mathrm{~min}$ ) using a chronograph. Only when the mouse hung passively and completely motionless, it was regarded as immobile. Mice that climbed their tails during the trials were excluded from data analysis.

Measurement of 5-HT and NE in hippocampal tissue. After the behavioral analyses, animals were given rest for 2 days. The levels of 5-HT and NE in the hippocampi of the experimental animals were determined by using 5-HT (F02611; Shanghai Westang Bio-Tech Co., Ltd., Shanghai, China) or NE ELISA kits (F16311; Shanghai Westang Bio-Tech Co., Ltd.). Protein quantitation was performed with a bicinchoninic acid kit (P0010S; Beyotime Institute of Biotechnology, Haimen, China).
Determination of pro-inflammatory cytokine levels by ELISA. The hippocampal levels of the pro-inflammatory cytokine IL-1 $\beta$ were detected with commercial ELISA kits (MLB00C; R\&D Systems, Minneapolis, MN, USA) following the manufacturer's protocols. The levels of IL- $1 \beta$ were expressed as pg/g total protein.

Western blot analysis. Western blot assays were performed according to a standard method. Hippocampus were extracted in RIPA buffer (consisting of a protease inhibitor cocktail) and protein concentration was quantified by BCA assay kit (Beyotime Institute of Biotechnology, Haimen, China). Total protein $(20-\mu \mathrm{g}$ aliquots) was separated by $10-15 \%$ SDS-PAGE with subsequent transfer onto polyvinylidene difluoride membranes (EMD Millipore, Billerica, MA, USA). After incubation with primary antibody to NLRP3 (1:1,000; 15101; Cell Signaling Technology Inc., Danvers, MA, USA), ASC (1:1,000; 67824; Cell Signaling Technology, Inc.), caspase-1 (1:500; ab1872; Abcam, Cambridge, UK) and GAPDH (1:1,000; 97166; Santa Cruz Biotechnology, Inc., Dallas, TX, USA) overnight at $4^{\circ} \mathrm{C}$, the blots were incubated with the secondary anti-rabbit $\mathrm{IgG}$ horseradish peroxidase (HRP)-linked antibody (1:1,000 for NLRP3 and ASC; 7074; Cell Signaling Technology Inc.), goat anti-rabbit IgG H\&L (HRP) (1:2,000 for caspase-1; ab205718; Abcam), anti-mouse IgG, HRP-linked antibody (1:2,000 for GAPDH; 7076; Santa Cruz Biotechnology, Inc.) for $1 \mathrm{~h}$ at room temperature. The immunoreactive bands were visualized with an enhanced chemiluminescence kit (20158; Thermo Fisher Scientific, Inc., Waltham, MA, USA). Images were captured with a Fuji LAS3000-mini imaging system (Fujifilm, Tokyo, Japan), and immunoreactive bands were quantified by Image J software (v1.41; NIH, Bethesda, MD, USA).

Statistical analysis. Data were analyzed with Graph Pad Prism 5.01 (GraphPad Software, Inc., La Jolla, CA, USA). Values are expressed as the mean \pm standard deviation as indicated. The significance between different groups was analyzed using one-way analysis of variance followed by Bonferroni's multiple comparisons test. $\mathrm{P}<0.05$ was considered to indicate a statistically significant difference.

\section{Results}

AT-I restores the decreased body weight in mice subjected to CUMS. As presented in Fig. 1, the body weight of mice in each group was similar at the beginning of the experiment. However, 3 weeks of CUMS significantly decreased the body weight compared with that of the control group $(\mathrm{P}<0.05)$. However, after 3 weeks of treatment, AT-I at all three doses as well as fluoxetine significantly restored the decreased body weight after CUMS.

AT-I alleviates CUMS-induced depressive-like behavior. To investigate the effect of AT-I in CUMS-induced depressive-like behavior, SFT (Fig. 2A), FST (Fig. 2B) and TST (Fig. 2C) were performed. As demonstrated in Fig. 2, the model group displayed a significant reduction in sucrose consumption in comparison with that in the control group, while treatment with AT-I and fluoxetine obviously increased the sucrose 


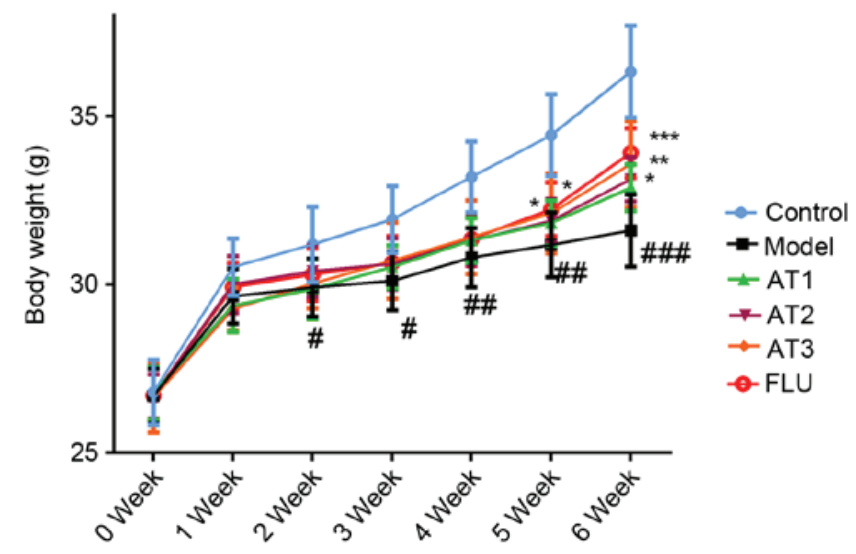

Figure 1. Effect of AT-I on body weight of mice. Values are expressed as the mean \pm standard deviation. ${ }^{\#} \mathrm{P}<0.05,{ }^{\# \#} \mathrm{P}<0.01$, ${ }^{\# \#} \mathrm{P}<0.001$ compared with the control group; ${ }^{*} \mathrm{P}<0.05,{ }^{* *} \mathrm{P}<0.01,{ }^{* * *} \mathrm{P}<0.001$ compared with the model group. AT1/2/3, model mice treated with atractylenolide-I at 5,10 or $20 \mathrm{mg} / \mathrm{kg}$; FLU, model mice treated with fluoxetine.

A

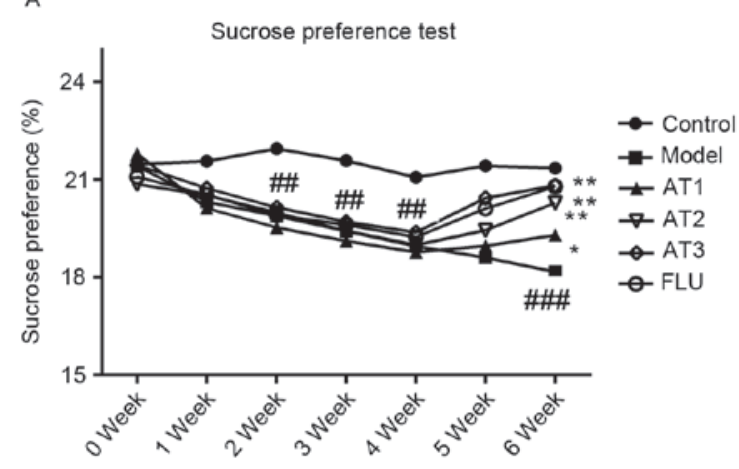

C

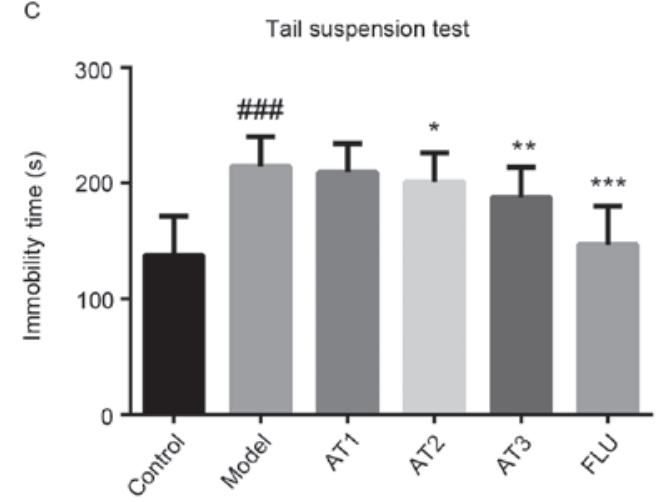

B

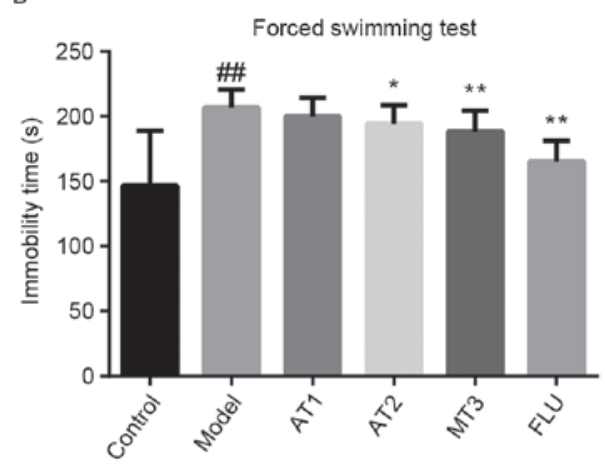

Figure 2. Effect of AT-I on chronic unpredictable mild stress-induced depressive-like behavior. (A) Sucrose preference test, (B) forced swimming test and (C) tail suspension test. Values are expressed as the mean \pm standard deviation $(\mathrm{n}=6) .{ }^{\# \#} \mathrm{P}<0.01,{ }^{\# \# \#} \mathrm{P}<0.001$ compared with the control group; ${ }^{*} \mathrm{P}<0.05,{ }^{* *} \mathrm{P}<0.01$, ${ }^{* * * *} \mathrm{P}<0.001$ compared with the model group. AT1/2/3, model mice treated with atractylenolide-I at $5,10 \mathrm{or} 20 \mathrm{mg} / \mathrm{kg}$; FLU, model mice treated with fluoxetine.

consumption in mice subjected to CUMS. As displayed in Fig. 2B and C, respectively, the immobility time in the FST and TST were significantly increased in this animal model, which was greatly reduced by treatment with AT-I or fluoxetine. All of these observations suggested that AT-I significantly alleviates CUMS-induced depression.

AT-I attenuates CUMS-induced imbalances in hippocampal neurotransmitter levels. As illustrated in Fig. 3A, compared with those in the control group, 5-HT levels in the hippocampus were obviously decreased after CUMS. However, treatment with AT-I or fluoxetine significantly increased hippocampal 5-HT levels compared with those in the model group. Furthermore, CUMS markedly reduced the NE levels in the hippocampi of mice, which was inhibited by treatment with AT-I and fluoxetine (Fig. 3B).

AT-I reduces CUMS-induced increases in hippocampal pro-inflammatory cytokine levels in mice. Next, the effect of AT-I on pro-inflammatory cytokine levels in the hippocampi 
A

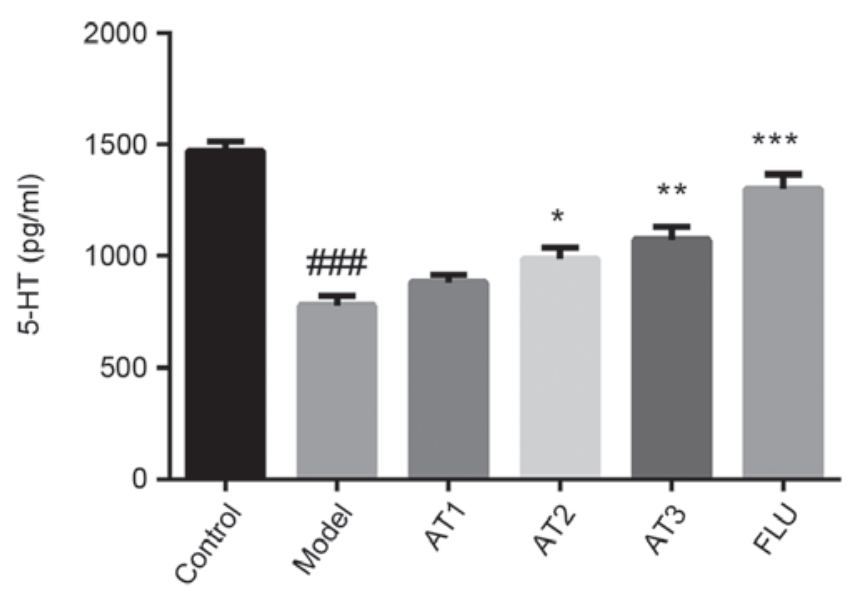

B

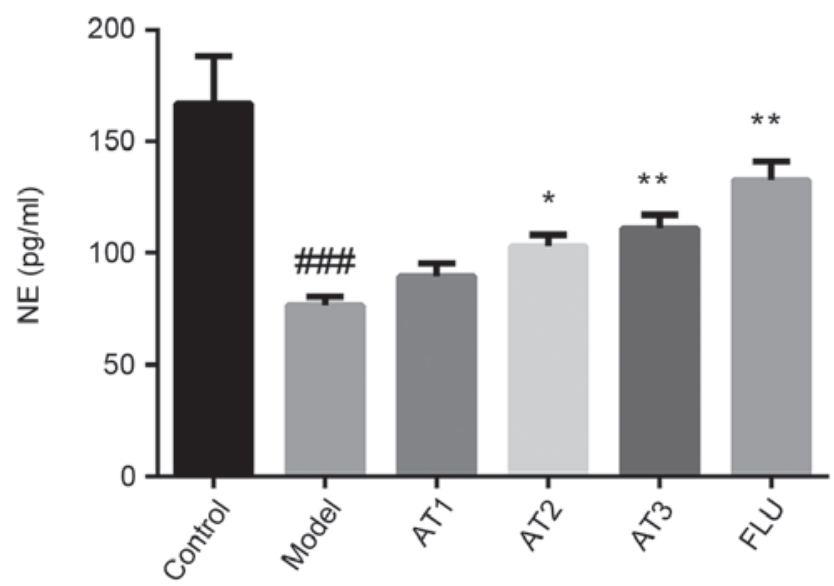

Figure 3. Effect of AT-I on hippocampal 5-HT and NE levels in chronic unpredictable mild stress-induced mice. (A) The levels of 5-HT in the hippocampus, (B) the levels of $\mathrm{NE}$ in the hippocampus. Values are expressed as the mean \pm standard deviation $(\mathrm{n}=6)$. ${ }^{\# \# "} \mathrm{P}<0.001$ compared with the control group; $\mathrm{P}<0.05$, ${ }^{* *} \mathrm{P}<0.01,{ }^{* * *} \mathrm{P}<0.001$ compared with the model group. AT1/2/3, model mice treated with atractylenolide-I at 5,10 or $20 \mathrm{mg} / \mathrm{kg}$; FLU, model mice treated with fluoxetine; 5-HT, serotonin; NE, norepinephrine.

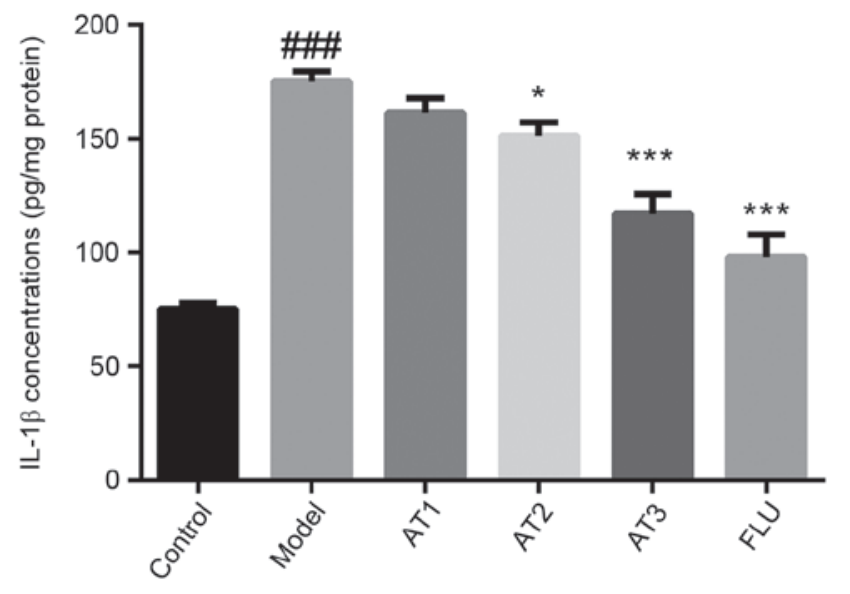

Figure 4. Effect of AT-I on hippocampal IL-1 $\beta$ concentrations in chronic unpredictable mild stress-induced mice. Values are expressed as the mean \pm standard deviation $(\mathrm{n}=6)$. ${ }^{\# \#} \mathrm{P}<0.001$ compared with the control group; ${ }^{*} \mathrm{P}<0.05,{ }^{* * *} \mathrm{P}<0.001$ compared with the model group. AT1/2/3, mode mice treated with atractylenolide-I at 5,10 or $20 \mathrm{mg} / \mathrm{kg}$; FLU, model mice treated with fluoxetine; IL, interleukin.

of mice subjected to CUMS was examined. The results revealed that CUMS significantly increased the hippocampal IL-1 $\beta$ concentration (Fig. 4). Of note, AT-I and fluoxetine treatment markedly reduced CUMS-induced hippocampal IL-1 $\beta$ concentrations in mice.

AT-I reduces CUMS-induced increases in the NLRP3 inflammasome in the hippocampi of mice. To investigate whether AT-I alleviated CUMS-induced depression via NLRP3, the expression of components of the inflammasome complex was detected by western blot analysis. As presented in Fig. 5, the hippocampal protein levels of NLRP3 and ASC were significantly upregulated by CUMS compared with those in the control group. Furthermore, the maturation of caspase-1 was markedly increased after CUMS in comparison with that in the control group. However, treatment with AT-I or fluoxetine significantly reduced the CUMS-induced activation of the NLRP3 inflammasome, as evidenced by a significant downregulation of NLRP3 and ASC protein expression as well as reduced maturation of caspase- 1 in the hippocampi of this animal model.

\section{Discussion}

The present study reported that AT-I treatment alleviated CUMS-induced depressive-like behavior and attenuated CUMS-associated decreases in hippocampal 5-HT and NE levels, increases in IL-1 $\beta$ levels and the activation of the NLRP3 inflammasome complex. To the best of our knowledge, the present study was the first to demonstrate the anti-depressive activity of AT-I in a mouse model of CUMS.

First, it was demonstrated that mice subjected to CUMS had a depressive-like behavior, including decreased sucrose preference percentages and longer immobility time in FST and TST. Of note, AT-I treatment significantly reduced these changes, as evidenced by increased sucrose preference as well as shortened immobility time in FST and TST. Of note, plenty of studies have confirmed that Xiaoyao San, a well-known traditional Chinese formula containing Rhizoma Atractylodis macrocephalae exerted anti-depressant-like effects in an animal model of CUMS-induced depression $(21,22)$ and perimenopausal women with depression (23). In addition, a study by Zhou et al (16) identified that AT-I may be the component of Xiaoyao San responsible for its anti-depressant effects. Therefore, the present study confirmed the anti-depressive activity of AT-I in a CUMS-induced animal model of depression. In addition, the present study observed that a 3-week CUMS procedure significantly reduced body weight gain compared with that in the control group, which is consistent with the result of a previous study (18).

Numerous studies have indicated that the neurotransmitters 5-HT and NE have an important role in learning and memory processes $(24,25)$. Therefore, their abnormal levels should provide an explanation for the cognitive deficits in depression. In accordance with these studies, the present 
A

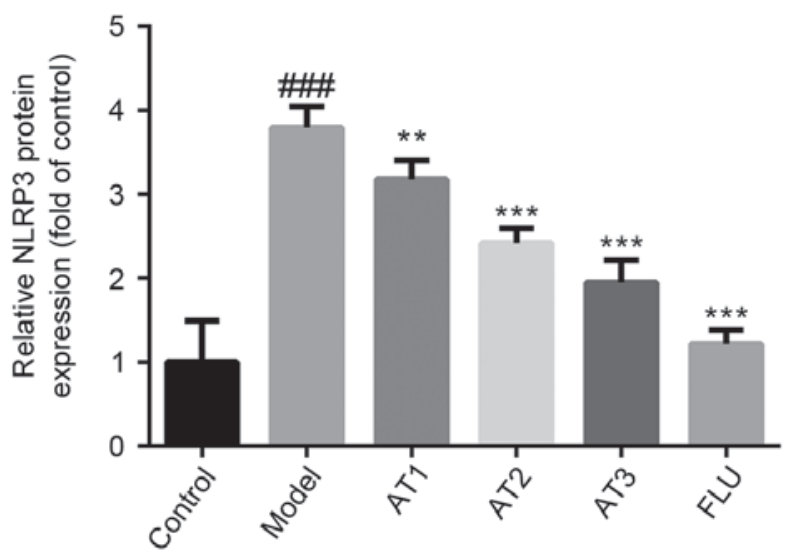

C

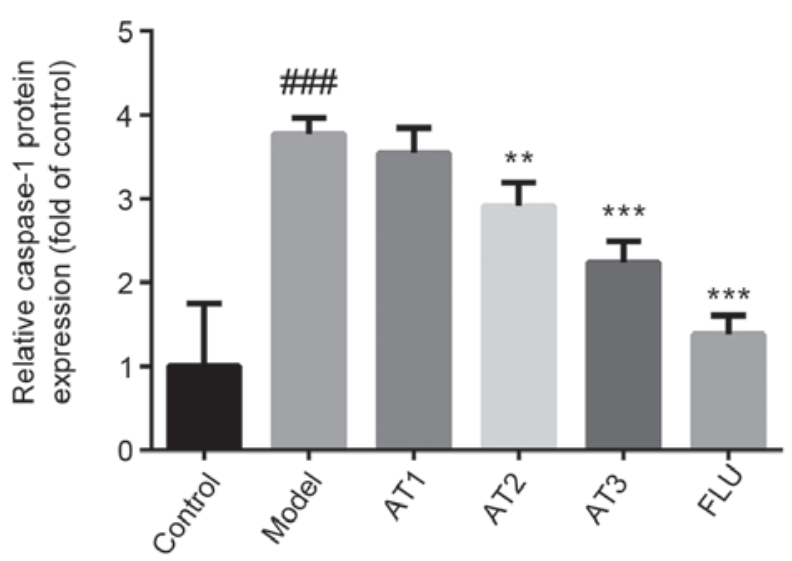

B

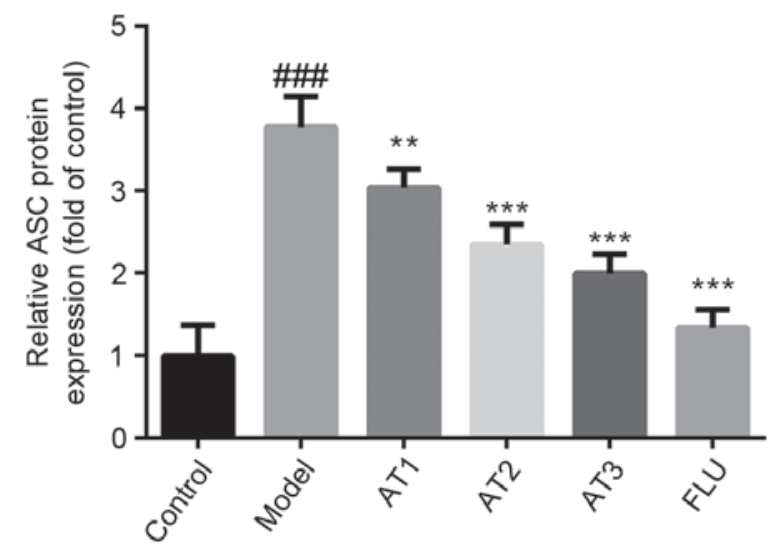

D
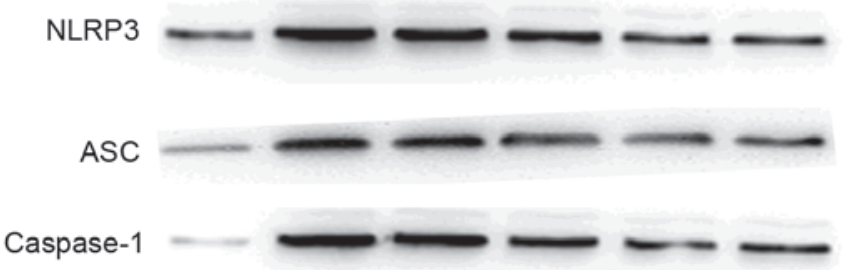

GAPDH

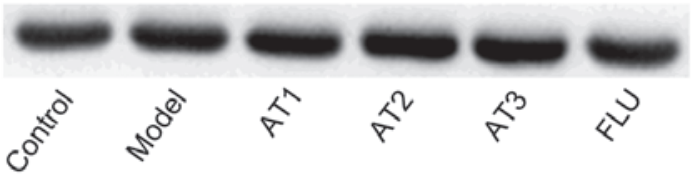

Figure 5. Effect of AT-I on hippocampal NLRP3 inflammasome complex protein levels in chronic unpredictable mild stress-induced mice. Relative expression of (A) NLRP3, (B) ASC and (C) caspase-1, and (D) representative western blot images. Values are expressed as the mean \pm standard deviation (n=6).

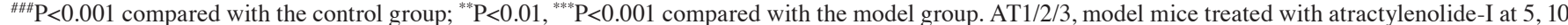
or $20 \mathrm{mg} / \mathrm{kg}$; FLU, model mice treated with fluoxetine; NLRP3, nucleotide binding and oligomerization domain-like receptor family pyrin domain-containing 3; ASC, apoptosis-associated speck-like protein.

study found that the levels of neurotransmitters 5-HT and NE were significantly decreased after the 3 -week CUMS procedure. However, treatment with AT-I for 3 weeks significantly restored CUMS-induced reduction of hippocampal 5-HT and NE levels in this animal model, implying that the monoamine system may be involved in the anti-depressant effect of AT-I.

Inflammatory cytokines have been reported to have a key role in the physiology and development of depression (26). While any possible aberrations of serum or peripheral IL-1 $\beta$ remain controversial (9), hippocampal IL-1 $\beta$ levels have been widely confirmed to be significantly increased under chronic stress $(27,28)$. Similarly, the present study found that a 3 -week CUMS procedure significantly elevated IL-1 $\beta$ in the hippocampi of mice. Of note, the elevation of hippocampal IL-1 $\beta$ levels in mice subjected to CUMS was partly reversed by treatment with AT-I for 3 weeks. Previous studies have indicated that AT-I exhibited anti-inflammatory activity and inhibited the production of IL-1 $\beta(15,29)$, which is consistent with the ELISA results for IL-1 $\beta$ of the present study.In addition, it was previously demonstrated that IL-1 $\beta$ activates the neurotransmitters 5-HT and NE in the CNS (30). Therefore, together with the results of the neurotransmitter assay, the results of the present study suggested that AT-I may ameliorate CUMS-induced depression in mice via inhibition of IL-1 $\beta$ production. Furthermore, the NLRP3 inflammasome, an important regulator of IL-1 $\beta$ transcription and function, has been proved to be involved in stress-induced depression (10). As observed in the present study, a 3-week CUMS procedure significantly activated the NLRP3 inflammasome, as evidenced by significantly increased NLRP3 and ASC protein levels as well as caspase-1 maturation, which is in agreement with a previous study (31). However, AT-I treatment for 3 weeks significantly suppressed the activation of the NLRP3 inflammasome complex in mice, suggesting that the anti-depressant effect of AT-I may alleviate CUMS-induced depression by inhibiting the activation of the NLRP3 inflammasome.

In conclusion, the results of the present study implied that AT-I exerts anti-depressive effects in a mouse model of CUMS-induced depression, and that the molecular mechanism may be associated with the inhibition of NLRP3 inflammasome activation. AT-I may be a potentially useful agent for the treatment of depression. 


\section{References}

1. Klinedinst NJ and Regenold WT: A mitochondrial bioenergetic basis of depression. J Bioenerg Biomembr 47: 155-171, 2015

2. Xue R, Jin ZL, Chen HX, Yuan L, He XH, Zhang YP, Meng YG, Xu JP, Zheng JQ, Zhong BH, et al: Antidepressant-like effects of $071031 \mathrm{~B}$, a novel serotonin and norepinephrine reuptake inhibitor. Eur Neuropsychopharmacol 23: 728-741, 2013.

3. Maes M: Depression is an inflammatory disease, but cell-mediated immune activation is the key component of depression. Prog Neuropsychopharmacol Biol Psychiatry 35: 664-675, 2011.

4. Adler UC, Marques AH and Calil HM: Inflammatory aspects of depression. Inflamm Allergy Drug Targets 7: 19-23, 2008.

5. Mikova O, Yakimova R, Bosmans E, Kenis G and Maes M Increased serum tumor necrosis factor alpha concentrations in major depression and multiple sclerosis. Eur Neuropsychopharmacol 11: 203-208, 2001.

6. Bluthé RM, Layé S, Michaud B, Combe C, Dantzer R and Parnet P: Role of interleukin-1beta and tumour necrosis factor-alpha in lipopolysaccharide-induced sickness behaviour: A study with interleukin-1 type I receptor-deficient mice. Eur J Neurosci 12: 4447-4456, 2000.

7. Franchi L, Eigenbrod T, Muñoz-Planillo R and Nuñez G: The inflammasome: A caspase-1-activation platform that regulates immune responses and disease pathogenesis. Nat Immunol 10 241-247, 2009

8. Alcocer-Gómez E, de Miguel M, Casas-Barquero N, Núñez-Vasco J, Sánchez-Alcazar JA, Fernández-Rodríguez A and Cordero MD: NLRP3 inflammasome is activated in mononuclear blood cells from patients with major depressive disorder. Brain Behav Immun 36: 111-117, 2014.

9. Pan Y, Chen XY, Zhang QY and Kong LD: Microglial NLRP3 inflammasome activation mediates IL-1 $\beta$-related inflammation in prefrontal cortex of depressive rats. Brain Behav Immun 41: 90-100, 2014

10. Zhang Y, Liu L, Liu YZ, Shen XL, Wu TY, Zhang T, Wang W, Wang YX and Jiang CL: NLRP3 inflammasome mediates chronic mild stress-induced depression in mice via neuroinflammation. Int J Neuropsychopharmacol 18: pii: pyv006, 2015.

11. Zhang JL, Huang WM and Zeng QY: Atractylenolide I protects mice from lipopolysaccharide-induced acute lung injury. Eur J Pharmacol 765: 94-99, 2015

12. Ji G, Chen R and Zheng J: Atractylenolide I inhibits lipopolysaccharide-induced inflammatory responses via mitogen-activated protein kinase pathways in RAW264.7 cells. Immunopharmacol Immunotoxicol 36: 420-425, 2014

13. Yu R, Yu BX, Chen JF, Lv XY, Yan ZJ, Cheng Y and Ma Q: Anti-tumor effects of Atractylenolide I on bladder cancer cells J Exp Clin Cancer Res 35: 40, 2016.

14. Huang JM, Zhang GN, Shi Y, Zha X, Zhu Y, Wang MM, Lin Q, Wang W, Lu HY, Ma SQ, et al: Atractylenolide-I sensitizes human ovarian cancer cells to paclitaxel by blocking activation of TLR4/MyD88-dependent pathway. Sci Rep 4: 3840, 2014.

15. Wang C, Duan H and He L: Inhibitory effect of atractylenolide I on angiogenesis in chronic inflammation in vivo and in vitro. Eur J Pharmacol 612: 143-152, 2009.

16. Zhou Y, Ren Y, Ma Z, Jia G, Gao X, Zhang L and Qin X: Identification and quantification of the major volatile constituents in antidepressant active fraction of xiaoyaosan by gas chromatography-mass spectrometry. J Ethnopharmacol 141: 187-192, 2012.
17. Deng XY, Li HY, Chen JJ, Li RP, Qu R, Fu Q and Ma SP: Thymol produces an antidepressant-like effect in a chronic unpredictable mild stress model of depression in mice. Behav Brain Res 291: 12-19, 2015.

18. Deng XY, Xue JS, Li HY, Ma ZQ, Fu Q, Qu R and Ma SP: Geraniol produces antidepressant-like effects in a chronic unpredictable mild stress mice model. Physiol Behav 152: 264-271, 2015.

19. Kurhe Y, Radhakrishnan M and Gupta D: Ondansetron attenuates depression co-morbid with obesity in obese mice subjected to chronic unpredictable mild stress; an approach using behavioral battery tests. Metab Brain Dis 29: 701-710, 2014.

20. Li M, Fu Q, Li Y, Li S, Xue J and Ma S: Emodin opposes chronic unpredictable mild stress induced depressive-like behavior in mice by upregulating the levels of hippocampal glucocorticoid receptor and brain-derived neurotrophic factor. Fitoterapia 98: $1-10,2014$.

21. Zhu X, Xia O, Han W, Shao M, Jing L, Fan Q, Liu Y, Diao J, Lv Z and Sun X: Xiao Yao San improves depressive-like behavior in rats through modulation of $\beta$-Arrestin 2-Mediated pathways in Hippocampus. Evid Based Complement Alternat Med 2014: 902516, 2014.

22. Ding XF, Zhao XH, Tao Y, Zhong WC, Fan Q, Diao JX, Liu YL, Chen YY, Chen JX and Lv ZP: Xiao Yao San improves depressive-like behaviors in rats with chronic immobilization stress through modulation of Locus Coeruleus-Norepinephrine system. Evid Based Complement Alternat Med 2014: 605914, 2014.

23. Du HG, Ming L, Chen SJ and Li CD: Xiaoyao pill for treatment of functional dyspepsia in perimenopausal women with depression. World J Gastroenterol 20: 16739-16744, 2014

24. Hou C, Jia F, Liu Y and Li L: CSF serotonin, 5-hydroxyindolacetic acid and neuropeptide $Y$ levels in severe major depressive disorder. Brain Res 1095: 154-158, 2006.

25. Kuhn M, Popovic A and Pezawas L: Neuroplasticity and memory formation in major depressive disorder: An imaging genetics perspective on serotonin and BDNF. Restor Neurol Neurosci 32: 25-49, 2014.

26. Anisman $\mathrm{H}$ and Merali Z: Cytokines, stress and depressive illness: Brain-immune interactions. Ann Med 35: 2-11, 2003.

27. Zhang Y, Su WJ, Chen Y, Wu TY, Gong H, Shen XL, Wang YX, Sun XJ and Jiang CL: Effects of hydrogen-rich water on depressive-like behavior in mice. Sci Rep 6: 23742, 2016.

28. Liu XL, Luo L, Liu BB, Li J, Geng D, Liu Q and Yi LT: Ethanol extracts from Hemerocallis citrina attenuate the upregulation of proinflammatory cytokines and indoleamine 2,3-dioxygenase in rats. J Ethnopharmacol 153: 484-490, 2014.

29. Wang A, Xiao Z, Zhou L, Zhang J, Li X and He Q: The protective effect of atractylenolide I on systemic inflammation in the mouse model of sepsis created by cecal ligation and puncture. Pharm Biol 54: 146-150, 2016.

30. Zhu CB, Blakely RD and Hewlett WA: The proinflammatory cytokines interleukin-1beta and tumor necrosis factor-alpha activate serotonin transporters. Neuropsychopharmacology 31: 2121-2131, 2006

31. Li R, Wang X, Qin T, Qu R and Ma S: Apigenin ameliorates chronic mild stress-induced depressive behavior by inhibiting interleukin-1 $\beta$ production and NLRP3 inflammasome activation in the rat brain. Behav Brain Res 296: 318-325, 2016. 